\title{
Simple Distances for Trajectories via Landmarks
}

\author{
Jeff M. Phillips \\ jeffp@cs.utah.edu \\ School of Computing, University of Utah. \\ Salt Lake City, Utah, USA
}

\author{
Pingfan Tang \\ tang1984@cs.utah.edu \\ School of Computing, University of Utah. \\ Salt Lake City, Utah, USA
}

\begin{abstract}
We develop a new class of distances for trajectories, based on the distance to a set of landmarks. These distances easily and interpretably map objects to a Euclidean space, are simple to compute, and perform well in data analysis tasks. For trajectories, they match and in some cases significantly out-perform all state-of-the-art other metrics, can effortlessly be used in $k$-means clustering, and directly plugged into approximate nearest neighbor approaches which immediately out-perform the best recent advances in trajectory similarity search by several orders of magnitude. These distances do not require complicated alignment (common in trajectory case). We show reasonable and often simple conditions under which these distances are metrics.
\end{abstract}

\section{CCS Concepts}

- Computing methodologies $\rightarrow$ Feature selection; • Theory of computation $\rightarrow$ Randomness, geometry and discrete structures.

\section{Keywords}

trajectory similarity, trajectory classification, sketching ${ }^{1}$

ACM Reference Format:

Jeff M. Phillips and Pingfan Tang . 2019. Simple Distances for Trajectories via Landmarks. In 27th ACM SIGSPATIAL International Conference on Advances in Geographic Information Systems (SIGSPATIAL '19), November 05-08, 2019, Chicago, IL, USA. ACM, New York, NY, USA, 4 pages. https://doi.org/10. $1145 / 3347146.3359098$

\section{Introduction}

The choice of a distance is often the most important modeling decision in any data analysis task. This choice is what determines which objects are close and which are far. However, this task is often taken lightly or made just based on what provides the simplest or easiest to compute option.

In this paper we explore what we believe to be a new and natural family of distances between trajectories. Our proposed distance $\mathrm{d}_{Q}$ uses a set $Q$ of landmark points, which could be points of interest for

\footnotetext{
${ }^{1}$ Thanks to NSF CCF-1350888, ACI-1443046, CNS- 1514520 , CNS-1564287, and IIS1816149. Part of the work was completed while visiting the Simons Institute for ToC.

Permission to make digital or hard copies of all or part of this work for personal or classroom use is granted without fee provided that copies are not made or distributed for profit or commercial advantage and that copies bear this notice and the full citation on the first page. Copyrights for components of this work owned by others than ACM must be honored. Abstracting with credit is permitted. To copy otherwise, or republish, to post on servers or to redistribute to lists, requires prior specific permission and/or a fee. Request permissions from permissions@acm.org.

SIGSPATIAL '19, November 05-08, 2019, Chicago, IL, USA

(C) 2019 Association for Computing Machinery.

ACM ISBN 978-1-4503-6909-1/19/11.

https://doi.org/10.1145/3347146.3359098
}

which a trajectory passing nearby has specific meaning. However, in a general case, $Q$ can be chosen as arbitrary or random points placed to cover a domain of focus. Then the new distances, instead of being directly between the objects themselves, are based on how they interact with the set of landmarks. In the simplest variant, for $n$ landmarks $Q$, for any object $J$ we create an $n$-dimensional vector $v_{J}=\left(v_{1}, v_{2}, \ldots, v_{n}\right)$ of the distance from $q_{i} \in Q$ to $J$, and the distance between two objects $J_{1}$ and $J_{2}$ is the Euclidean distance between the vectors $\left\|v_{J_{1}}-v_{J_{2}}\right\|$. In other words, we vectorize the distance between complex objects.

In Section 2, we introduce two intuitive variants $\mathrm{d}_{Q}$ and $\mathrm{d}_{Q}^{\pi}$ of our new distance; these are psuedometrics, and for dense enough $Q$ are sometimes metrics. We demonstrate that they are at the least as effective for classification tasks (via KNN classifiers) as compared to the best of 8 other common metrics, and in some cases significantly outperforms all of these measures. Moreover, the previous competing variants are typically significantly more complicated or computationally intensive, and may require parameter tuning.

In contrast to most of these trajectory distance alternatives, all of our proposed distances are very simple to compute and work with. They map curves to a $|Q|$-dimensional parameter space where Euclidean distance (or similar) is used. In $\mathrm{d}_{Q}$ for curves, each coordinate $v_{i}$ is the distance to the closest point on the curve from $q_{i} \in Q$. In $\mathrm{d}_{Q}^{\pi}$ each "coordinate" is actually the $d$ coordinates of the closest point on the curve (not just the distance). These mappings are effective with only 10 or 20 landmark points $Q$. And because they have a familiar Euclidean structure, we can immediately invoke favorite algorithms in this space, from Lloyds for $k$-means clustering, linear and kernel SVM to highly-engineered approximate nearest neighbor libraries. In comparison to recent trajectory similarity search systems $[12,15]$, we show using $\mathrm{d}_{Q}$ is much simpler and several orders of magnitude faster.

In summary, this paper introduces a family of metrics for trajectories which are incredibly simple to use, provide a sketch vector in Euclidean space, have many other desirable mathematical properties, and perform as well as and often significantly better than any existing measure. Many details are deferred to full version [10].

\section{Landmark Distances Between Trajectories}

We define two variants of $\mathrm{d}_{Q}$ for trajectories, focused on their modeling as piecewise-linear curves on $\mathbb{R}^{2}$. Let $\Gamma$ define the set of such curves, and they are specified by a series of critical points $\left\langle c_{0}, c_{1}, \ldots, c_{k}\right\rangle$. The curve $\gamma \in \Gamma$ is the subset of $\mathbb{R}^{2}$ defined by the $k$ segments $s_{1}, s_{2}, \ldots, s_{k}$ where $s_{i}=c_{i-1} c_{i}$ is the continuous set of points between critical points $c_{i-1}$ and $c_{i}$. For notational convenience, we will describe curves with $k$ segments, but the distance will not require this. Moreover, since we model the trajectory as a 


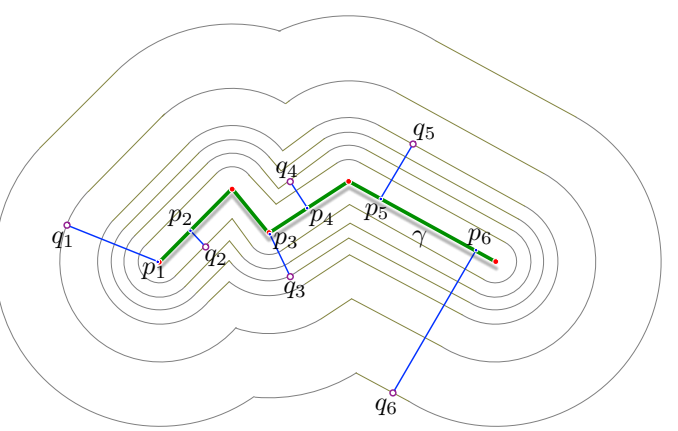

Figure 1: Illustrating $q_{i}$ and $p_{i}$ on a trajectory for $\mathrm{d}_{Q}$ and $\mathrm{d}_{Q}^{\pi}$.

subset of $\mathbb{R}^{2}$, it will not distinguish trajectories of different speeds or moving in opposite directions but following the same paths.

Now for a curve $\gamma \in \Gamma$ and size $n$ point set $Q \subset \mathbb{R}^{2}$, define $v_{i}=\min _{p \in \gamma}\left\|q_{i}-p\right\|$ and $p_{i}=\arg \min _{p \in \gamma}\left\|q_{i}-p\right\|$; see Figure 1. For two curves $\gamma^{(1)}$ and $\gamma^{(2)}$ denote these values as $v_{i}^{(1)}, p_{i}^{(1)}$ and $v_{i}^{(2)}, p_{i}^{(2)}$ respectively. Our distances are then defined as:

$$
\begin{aligned}
& \mathrm{d}_{Q}\left(\gamma^{(1)}, \gamma^{(2)}\right)=\left(\frac{1}{n} \sum_{i=1}^{n}\left(v_{i}^{(1)}-v_{i}^{(2)}\right)^{2}\right)^{\frac{1}{2}}, \\
& \mathrm{~d}_{Q}^{\pi}\left(\gamma^{(1)}, \gamma^{(2)}\right)=\frac{1}{n} \sum_{i=1}^{n}\left(\left\|p_{i}^{(1)}-p_{i}^{(2)}\right\|\right) .
\end{aligned}
$$

The second variant $\mathrm{d}_{Q}^{\pi}$ (the projected landmark distance) projects $Q$ onto the closest points of the curves, and then computes the average distances with respect to these projected points. Both variants are psuedometrics because of their Euclidean representation, and the full version [10] shows that if $Q$ is dense enough, they are full metrics.

\section{Trajectories Analysis via New Distances}

We demonstrate that $\mathrm{d}_{Q}$ and $\mathrm{d}_{Q}^{\pi}$ work effectively on real world problems. These approaches achieve state-of-the-art performance, are incredibly simple to use, and their sketched representation plugs directly into $k$-means clustering, KNN or SVM classifiers, or ANN libraries. We show that only a small number of landmarks are needed for good accuracy.

\subsection{Related Trajectory Distances, and Landmarks}

There are by now numerous definitions of trajectories, with a variety of different aspects they can model and take into account.

We compare the classification errors found using $\mathrm{d}_{Q}$ and $\mathrm{d}_{Q}^{\pi}$ with a series of representative distances for trajectories. These are: Euclidean distance among the critical points (Eu) [17], discrete Frechet distance $(\mathrm{dF})$ [4], dynamic time warping distance (DTW) [16], discrete Hausdorff distance ( $\mathrm{dH})$ [9], longest common subsequence distance (LCSS) [13], edit distance for real sequences (EDR) [2]. We also compare against the locality sensitive hashing distance $\left(\mathrm{LSH}_{Q}\right)$, and its ordered variant $\left(\mathrm{LSH}_{Q}\right)$ [1], which consider the intersection of the trajectories with a set of disks. This is conceptually similar to our methods, where we can think of the landmarks $Q$ as the centers of disks (as we do in experiments); their approach has

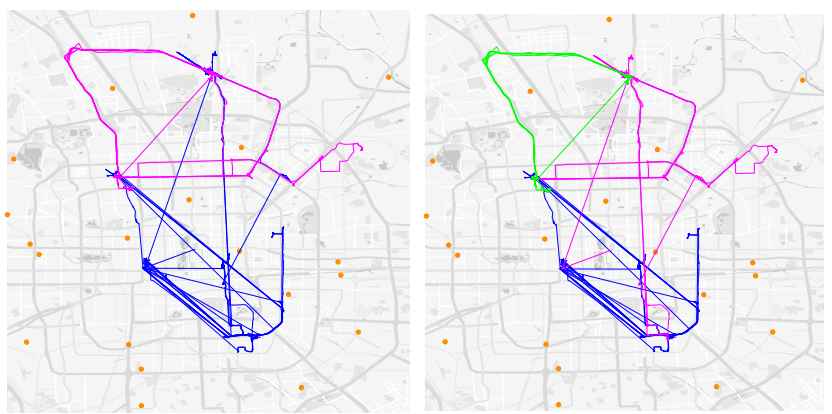

Figure 2: 2 or 3 clusters (color-coded) under $k$-means on $\mathrm{d}_{Q}$ with 20 landmarks $Q$ shown overlaid on Beijing.

a radius parameter $r$, and is not a metric. For LCSS, EDR, $\mathrm{LSH} 1_{Q}$, and $\mathrm{LSH}_{2} Q$ we tried various parameters and report the best.

Even beyond the recent trajectory LSH paper [1], the use of waypoints to provide a distance between trajectories is not new. However, they are typically used in other contexts, such as annotating with geolocated social media [14]. Or for instance, in the context of a line of work [6-8] seeking to find the $k$ nearest timeencoded trajectories to a given point at a specific time, Lin et al. [8] use a set of landmarks $Q$ to map trajectories and query points into the Voronoi cells of $Q$ to quickly help in pruning.

\subsection{Warm-up: $k$-means Clustering}

As a warm up, we consider clustering the 42 trajectories from user $i d_{155}$ in the Geolife GPS trajectory dataset [18]. We randomly choose 20 spread-out Beijing POIs as the landmark set $Q$, shown as orange dots in Figure 2. Using $\mathrm{d}_{Q}$, this maps each trajectory $\gamma$ to $\mathbb{R}^{20}$, and we directly run Lloyd's algorithm for $k$-means clustering with $k=2,3$, and color-code the corresponding trajectories in Figure 2. We observe that although the trajectories are intertwined, there is a central-city cluster found in both cases, and either 1 or 2 clusters found on the north side.

\subsection{Classifying Trajectories 1: Beijing Drivers}

We also consider classifying trajectories from users in the Geolife dataset [18] with the same 20 POI landmarks $Q$ as in the clustering example. There are 182 users, and each user has several trajectories in Beijing. We only consider those trajectories with more than 10 critical points, and if a user has less than 10 such trajectories, then we remove this user. Thus, 54 users are removed, and in the remaining 128 users, 20 of them have more than 200 trajectories. For each of these users, we just randomly sample 200 trajectories (without replacement), to avoid severe imbalance in classification.

Consider two sets of trajectories $\Gamma^{(1)}$ and $\Gamma^{(2)}$. Letting $\left|\Gamma^{(1)}\right|=m_{1}$ and $\left|\Gamma^{(2)}\right|=m_{2}$, we randomly sample $\left\lfloor\frac{3 m_{1}}{10}\right\rfloor$ trajectories from $\Gamma^{(1)}$ and $\left\lfloor\frac{3 m_{2}}{10}\right\rfloor$ trajectories from $\Gamma^{(2)}$ respectively to form a test set, and use the other trajectories in $\Gamma^{(1)} \cup \Gamma^{(2)}$ as the training data. Then we choose an algorithm and metric to do classification, and compute the error. For users with $i d_{1}$ and $i d_{2}$, we do this 10 times and take the mean error as error $\left(i d_{1}, i d_{2}\right)$. We compute error $\left(i d_{1}, i d_{2}\right)$ for all 8128 pairs of 128 uses, and then output the mean, median, and variance of these 8128 errors. 
Table 1: Classification error on Beijing Drivers with KNN.

\begin{tabular}{r|cccccccccc}
\hline & $\mathrm{d}_{Q}$ & $\mathrm{~d}_{Q}^{\pi}$ & $\mathrm{Eu}$ & $\mathrm{dF}$ & $\mathrm{DTW}$ & $\mathrm{dH}$ & $\mathrm{LCSS}$ & $\mathrm{EDR}$ & $\mathrm{LSH}_{Q} \mathrm{LSH}_{Q}$ \\
best param & - & - & - & - & - & - & $\varepsilon=0.005, \delta=10$ & $\varepsilon=0.005$ & $r=0.06$ & $r=0.1$ \\
\hline mean & 0.0817 & 0.0724 & 0.0811 & 0.1045 & 0.0722 & 0.0883 & 0.0714 & 0.0802 & 0.1290 & 0.2409 \\
median & 0.0667 & 0.0581 & 0.0654 & 0.0873 & 0.0571 & 0.0722 & 0.0500 & 0.0554 & 0.0949 & 0.2182 \\
variance & 0.0039 & 0.0033 & 0.0040 & 0.0054 & 0.0036 & 0.0043 & 0.0054 & 0.0070 & 0.0128 & 0.0210 \\
\hline
\end{tabular}

Table 2: Classification error on Beijing extended.

\begin{tabular}{r|ccccccc}
\hline statistic & $\mathrm{d}_{Q}$ & $\mathrm{~d}_{Q}^{\pi}$ & Eu & $\mathrm{d}_{Q}$ & $\mathrm{~d}_{Q}^{\pi}$ & $\mathrm{d}_{Q}$ & $\mathrm{~d}_{Q}^{\pi}$ \\
$|Q|$ & 20 & 20 & 20 & 200 & 200 & 200 & 200 \\
classifier & SVM & SVM & SVM & SVM & SVM & KNN & KNN \\
\hline mean & 0.0727 & 0.0733 & 0.0845 & 0.0722 & 0.0726 & 0.0801 & $\mathbf{0 . 0 7 0 8}$ \\
median & 0.0587 & 0.0588 & 0.0690 & 0.0581 & 0.0583 & 0.0650 & 0.0558 \\
variance & 0.0035 & 0.0036 & 0.0045 & 0.0035 & 0.0036 & 0.0038 & 0.0033 \\
\hline
\end{tabular}

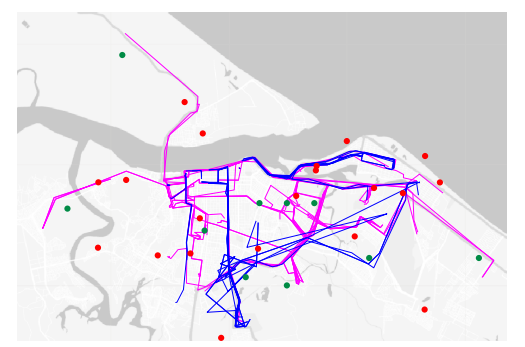

For all of these 10 distances, we use the KNN classification $(K=$ 5); see Table 1 . The lowest error rates of about $7 \%$ error is achieved by $\mathrm{d}_{Q}^{\pi}$, DTW and LCSS; they are within the variance bounds of each other. Then $\mathrm{d}_{Q}, \mathrm{Eu}$, and EDR achieve error about $8 \%$, again within the error bounds of each other. Other metrics perform worse with for example, $\mathrm{dF}$ at $10 \%, \mathrm{LSH}_{Q}$ at $13 \%$, and $\mathrm{LSH}_{2} Q$ at $24 \%$ error.

For $\mathrm{d}_{Q}, \mathrm{~d}_{Q}^{\pi}$ and $\mathrm{Eu}$, since they map a trajectory to a vector in Euclidean space, we can also directly use SVM to classify these vectors. From Table 2, we can see for SVM (with Gaussian kernel), both $\mathrm{d}_{Q}$ and $\mathrm{d}_{Q}^{\pi}$ are better than Eu. Both $\mathrm{d}_{Q}$ and $\mathrm{d}_{Q}^{\pi}$ achieve an error rate of about $7 \%$ which is less than the about $8 \%$ by Eu.

As we increase the size of $Q$ to 200 (chosen at random), then both $\mathrm{d}_{Q}$ and $\mathrm{d}_{Q}^{\pi}$ slightly improve in performance, but not drastically. The error statistics is shown in Table 2, from which we can see for $\mathrm{KNN}$, the performance of $\mathrm{d}_{Q}$ is better than Euclidean distance, and $\mathrm{d}_{Q}^{\pi}$ provides the smallest error (mean error 0.0708 , smaller than 0.0714 of LCSS). Moreover, we can see as $|Q|$ increases, the error of $\mathrm{d}_{Q}$ and $\mathrm{d}_{Q}^{\pi}$ also decrease. The relatively small improvement also demonstrates that even with a small size, random $Q$, the distances still perform at or near the state-of-the-art.

\subsection{Classifying Trajectories 2: Bus versus Car}

As another example, we consider the GPS Trajectories Data Set [3] in UCI machine learning repository. There are 87 car trajectories, and 76 bus trajectories in Aracaju, a city of Brazil. We remove those trajectories having less than 10 critical points, and then 78 car trajectories and 45 bus trajectories are left. These 123 trajectories are shown in Figure 3, where pink curves are cars and blue curves are busses. We hand-pick 10 points as $Q_{1}$ such that each point is close to one class of trajectories, and randomly generate 20 points as $Q_{2}$. Each time we randomly choose 23 car trajectories and 13 bus trajectories as test data, and use other trajectories as training data to perform classification experiments, and compute the error. We do this 1000 times to compute mean, median and variance.

The results are shown in Table 3, and we see the KNN classification results using all 14 distance, using either $Q_{1}$ (10 chosen near data) or $Q_{2}$ (20 randomly chosen). The results are slightly better for $Q_{2}$ in almost all distances $\mathrm{d}_{Q}, \mathrm{~d}_{Q}^{\pi}, \mathrm{LSH}_{Q}$, and $\mathrm{LSH}_{Q}$.
Figure 3: Bus (blue) and car (pink) trajectories with landmark sets $Q_{1}$ (green points), $Q_{2}$ (red points).

In these experiments on $Q_{2}$, the best mean error (about $21 \%$ to $22 \%$ ) is achieved by $\mathrm{d}_{Q}$, and $\mathrm{LSH}_{Q}$ (which required a parameter search). While $\mathrm{d}_{Q}^{\pi}$, LCSS, EDR, and $\mathrm{LSH}_{Q}$ achieve error between $25 \%$ and $27 \%$. Noticeably, the methods which were competitive with $\mathrm{d}_{Q}$ and $\mathrm{d}_{Q}^{\pi}$ on the Beijing Drivers data are EDR, which required a parameter tuned, as well as DTW and Eu, which now have error rate above $31 \%$. As a baseline, always predicting "car" obtains $36 \%$ error.

We show the results of applying Gaussian SVM in Table 3. Again the difference is small between $Q_{2}$ and $Q_{1}$ where for $\mathrm{d}_{Q}$ and $\mathrm{d}_{Q}^{\pi}$ the mean error is only $16 \%$ to $20 \%$. The overall best is $\mathrm{d}_{Q_{1}}^{\pi}$ achieving a mean error of $16.59 \%$.

Table 3: Classification error on Bus vs. Car.

\begin{tabular}{|c|c|c|c|c|}
\hline & distance & mean & median & variance \\
\hline \multirow{14}{*}{$\mathrm{KNN}$} & $\mathrm{d}_{Q_{1}}$ & 0.2331 & 0.2222 & 0.0045 \\
\hline & $\mathrm{d}_{Q_{2}}$ & 0.2229 & 0.2222 & 0.0041 \\
\hline & $\mathrm{d}_{Q_{1}}^{\pi / 2}$ & 0.2608 & 0.2500 & 0.0039 \\
\hline & $\mathrm{d}_{Q_{2}}^{\tilde{\pi}}$ & 0.2505 & 0.2500 & 0.0039 \\
\hline & $\mathrm{Eu}$ & 0.3323 & 0.3333 & 0.0044 \\
\hline & $\mathrm{dF}$ & 0.3431 & 0.3333 & 0.0045 \\
\hline & DTW & 0.3118 & 0.3056 & 0.0046 \\
\hline & $\mathrm{dH}$ & 0.3284 & 0.3333 & 0.0039 \\
\hline & $\operatorname{CSS}(\varepsilon=0.015, \delta=3)$ & 0.2448 & 0.2500 & 0.0037 \\
\hline & $\operatorname{EDR}(\varepsilon=0.015)$ & 0.2640 & 0.2500 & 0.0039 \\
\hline & $\mathrm{LSH}_{1}{ }_{Q_{1}}(r=0.02)$ & 0.2673 & 0.2778 & 0.0020 \\
\hline & $\mathrm{LSH} 2 Q_{Q_{1}}(r=0.08)$ & 0.2516 & 0.2500 & 0.0022 \\
\hline & $\mathrm{LSH}_{1}{ }_{Q_{2}}(r=0.03)$ & 0.2209 & 0.2222 & 0.0039 \\
\hline & $\mathrm{LSH} 2 Q_{2}(r=0.05)$ & 0.2690 & 0.2778 & 0.0022 \\
\hline \multirow{5}{*}{ Gaussian SVM } & $\mathrm{Eu}$ & 0.2239 & 0.2222 & 0.0034 \\
\hline & $\mathrm{d}_{Q_{1}}$ & 0.1894 & 0.1944 & 0.0029 \\
\hline & $\mathrm{d}_{Q_{2}}$ & 0.1968 & 0.1944 & 0.0033 \\
\hline & $\mathrm{d}_{O_{1}}^{\pi}$ & 0.1659 & 0.1667 & 0.0033 \\
\hline & $\mathrm{d}_{Q_{2}}^{\tilde{\pi}^{1}}$ & 0.1731 & 0.1667 & 0.0033 \\
\hline
\end{tabular}


Table 4: The running time experiment of KNN search.

\begin{tabular}{r|cccccc}
\hline$|Q|$ & 12 & 20 & 28 & 36 & 44 & 52 \\
\hline preprocessing time (s) & 38 & 62 & 88 & 114 & 138 & 160 \\
sketch size (MB) & 337 & 560 & 785 & 1012 & 1331 & 1536 \\
index time (s) & 106 & 109 & 114 & 119 & 124 & 129 \\
index file size (MB) & 999 & 999 & 1005 & 1002 & 1007 & 1001 \\
query time $\left(10^{-4} \mathrm{~s}\right)$ & 4.2 & 3.7 & 4.2 & 3.2 & 3.5 & 3.7 \\
\hline
\end{tabular}

\subsection{Using $\mathrm{d}_{Q}$ in Nearest Neighbor Search}

We demonstrate that $\mathrm{d}_{Q}$ 's sketched representation of the trajectories in $\mathbb{R}^{|Q|}$ allows for extremely efficient $k$-nearest neighbor search. We consider two representative methods $[12,15]$ for comparison; but do all, (e.g., [5]) which require timing information.

As a first comparison, consider a recent heavily-optimized kNN search algorithm focusing on Hausdorff and $\mathrm{dF}$ distances [15]; this system, DFT, is optimized for distributed algorithms on a cluster, but show results on 1 node which we compare against. We obtained a random sample of the GEN-TRAJ data set containing $m=3$ million trajectories, using 36GB of storage (larger than their 30.9GB dataset [15]). From their Figure 10, their indexes take 2000 to 6000 sec to build, and kNN queries require 50 to 200 seconds for $k=10$.

Another distributed system DITA [12] for trajectory similarity search focuses on DTW, returning all trajectories within a threshold. In their [12] Figures 7(a) and 8(a), using 256 cores they achieve query time between 0.001 and 0.01 seconds on Beijing (10.4GB) and Chengdu (28GB) datasets.

To perform $\mathrm{kNN}$ queries using $\mathrm{d}_{Q}$ we can sketch trajectories as $|Q|$-dimensional vectors and use Euclidean distance. Hence, once we create the sketches, we can use any of the highly optimized packages for kNN Euclidean queries (c.f., http://ann-benchmarks.com); we choose a consistent top performer K-Graph (https://github.com/ aaalgo/kgraph) with settings: recall $=0.99$ and max_iteration $=50$. We run on a desktop with a 6-core Intel Xeon CPU ES-1650 v3 @3.5GHz processor, and 128GB RAM; the same processor as in DFT [15].

We randomly choose a set of landmarks among the trajectories with $|Q|=\{12,20,28,36,44,52\}$. From these $Q$ we preprocess the data to derive $m \times|Q|$ sketches, a txt file we pass to K-Graph. Then $\mathrm{K}$-Graph builds an index, and allows queries. The preprocessing time (to build sketch), sketch file size, time to build K-Graph's index, that index size, and the average query time are shown in Table 4.

The preprocessing and index building times take 38 to $160 \mathrm{sec}-$ onds and 106 to 129 seconds, respectively. By comparison, it takes 673 seconds to load the raw data into memory. Combined they are an order of magnitude faster than the index build time for Hausdorff in DFT [15]. The sketch size is only 300 to $1500 \mathrm{MB}$, and the index sizes are $1000 \mathrm{MB}$; reducing the size by 1 or 2 orders of magnitude from the original size. Finally, the query times are only 0.00032 to 0.00042 seconds; that is 5 orders of magnitude faster than the DFT index optimized for Hausdorff distance! and 1 to 2 orders of magnitude faster than DITA optimized for DTW and using 256 cores on smaller data. Thus, using $\mathrm{d}_{Q}$ (and existing libraries) allows for small data sketches, and extremely efficient kNN queries.

\section{Conclusion and Discussion}

We introduce two landmark-based distances $\mathrm{d}_{Q}$ and $\mathrm{d}_{Q}^{\pi}$, with applications to trajectories. These have nice mathematical properties,
Table 5: Distances on analysis tasks as: best $\bullet$, competitive -, near competitive $\circ$; possible $\checkmark$ or possible but slower $\checkmark$.

\begin{tabular}{r|ccccccccc}
\hline task & $\mathrm{d}_{Q}$ & $\mathrm{~d}_{Q}^{\pi}$ & Eu & $\mathrm{dF}$ & DTW & dH & LCSS & EDR & LSH $_{Q}$ \\
\hline easy clust & $\checkmark$ & $\checkmark$ & $\checkmark$ & - & - & - & - & - & - \\
learn 1 & $\bullet$ & $\bullet$ & $\bullet$ & $\circ$ & $\bullet$ & $\bullet$ & $\bullet$ & $\bullet$ & - \\
learn 2 & $\bullet$ & $\bullet$ & $\circ$ & - & $\bullet$ & - & $\circ$ & $\bullet$ & $\circ$ \\
fast NN & $\checkmark$ & $\checkmark$ & $\checkmark$ & - & $\checkmark$ & - & - & - & $\checkmark$ \\
\hline any $k$ & $\checkmark$ & $\checkmark$ & - & $\checkmark$ & $\checkmark$ & $\checkmark$ & $\checkmark$ & $\checkmark$ & $\checkmark$ \\
\hline
\end{tabular}

e.g., being psuedo-metrics, metrics. On trajectories, new metrics $\mathrm{d}_{Q}$ and $\mathrm{d}_{Q}^{\pi}$ are the most general and best or competitive against all other distances in all analysis tasks; see Table 5.

The landmark set $Q$ can be randomly chosen and small (20 is often sufficient), or its points can hold specific meaning in which case, the interpretation and discriminatory ability of the distances are greatly enhanced. A companion paper [11] provides an in depth theoretical study of how many landmarks are required to preserve certain errors, how to chose them, and when curves can be explicitly recovered from them.

These provide meaningful vectorized representations. They are general and simple to compute and work with.

Software. Code for reproducing experiments in Section 3 is available here https://drive.google.com/open?id=1Z_Na1nfioM_We8b1F nTU5UVuOYCjbP-j

\section{References}

[1] Maria Astefanoaei, Paul Cesaretti, Panagiota Katsikouli, and Mayank Goswami andRik Sarkar. 2018. Multi-resolution sketches and locality sensitive hashing for fast trajectory processing. In SIGSPATIAL.

[2] Lei Chen, , M. Tamer Özsu, and Vincent Oria. 2005. Robust and fast similarity search for moving object trajectories.. In SIGMOD.

[3] Michael O. Cruz, Hendrik Macedo, R. Barreto, and Adolfo Guimaraes. 2016. GPS Trajectories Data Set. https://archive.ics.uci.edu/ml/datasets/GPS+Trajectories

[4] Thomas Eiter and Heikki Mannila. 1994. Computing Discrete Frechet Distance. Technical Report. Christian Doppler Laboratory for Expert Systems.

[5] Yixiang Fang, Reynold Cheng, Wenbin Tang, Silviu Maniu, and Xuan S. Yang:. 2016. Scalable algorithms for nearest-neighbor joins on big trajectory data. In ICDE.

[6] Elias Frentzos, Kostas Gratsias, Nikos Pelekis, and Yannis Theodoridis. 2005. Nearest Neighbor Search on Moving Object Trajectories. In SSTD.

[7] Ralf Hartmut Güting, Thomas Behr, and Jianqiu Xu. 2010. Efficient k-nearest neighbor search on moving object trajectories. In $V L D B$.

[8] Dan Lin, Rui Zhang, and Aoying Zhou. 2006. Indexing Fast Moving Objects for kNN Queries Based on Nearest Landmarks. GeoInformatica 10 (2006), 423-445.

[9] Facundo Mémoli. 2008. Gromov-Hausdorff distances in Euclidean spaces. Nonrigid shape analysis and deformable image registration Workshop (2008).

[10] Jeff M. Phillips and Pingfan Tang. 2019. Simple Distances for Trajectories via Landmarks. Technical Report. arXiv: 1804.11284.

[11] Jeff M. Phillips and Pingfan Tang. 2019. Sketched MinDist. Technical Report. arXiv: 1907.02171.

[12] Zeyuan Shang, Guoliang Li, and Zhifeng Bao. 2018. DITA: Distributed in-memory trajectory analytics. In SIGMOD.

[13] Michail Vlachos, George Kollios, and Dimitrios Gunopulos. 2002. Discovering similar multidimensional trajectories. In ICDE.

[14] Fei Wu, Zhenhui Li, Wang-Chien Lee, Hongjian Wang, and Zhuojie Huang. 2015. Semantic Annotation of Mobility Data using Social Media. In WWW.

[15] Dong Xie, Feifei Li, and Jeff M. Phillips. 2017. Distributed trajectory similarity search. In $V L D B$.

[16] Byoung-Kee Yi, H.V. Jagadish, and Christos Faloutsos. 1998. Efficient retrieval of similar time sequences under time warping. In ICDE.

[17] Zhang Zhang, Kaiqi Huang, and Tieniu Tan. 2006. Comparison of similarity measures for trajectory clustering in outdoor surveillance scenes. In ICPR.

[18] Yu Zheng, Hao Fu, Xing Xie, Wei-Ying Ma, and Quannan Li. 2011. Geolife GPS trajectory dataset - User Guide. https://www.microsoft.com/en-us/research/ publication/geolife-gps-trajectory-dataset-user-guide/ 